\title{
Maximum likelihood state between measurements
}

\author{
Alfredo Luis* \\ Departamento de Óptica, Facultad de Ciencias Físicas, Universidad Complutense, 28040 Madrid, Spain
}

(Received 9 May 2009; published 2 September 2009)

\begin{abstract}
We estimate the quantum state of a system between two measurements via a maximum likelihood strategy. This incorporates all the information provided by preselection and postselection in a legitimate quantum state. Moreover, it allows to include any other constraint derived from practical considerations.

DOI: 10.1103/PhysRevA.80.034101

PACS number(s): 03.65.Ca, 03.65.Wj, 03.67.- a, 42.50.Dv
\end{abstract}

\section{INTRODUCTION}

The peculiarities of quantum measurement have attracted a lot of attention since the beginning of the quantum theory. The behavior of system properties before and after measurements has given rise to many paradoxes highlighting relevant features of the quantum theory.

In this work we address what can be said about a quantum system between two measurements. This is to say between the preparation of the initial state and the observation of the final state [1-3]. These two states, referred to as preselected and postselected states, are fixed and represent the information about the system.

In this work we apply a maximum likelihood strategy to estimate the state of the system between measurements embodying all information. This is done by maximizing the probability of obtaining the observed outcomes in the preselection and postselection measurements (Sec. II).

The main properties of this approach are as follows: (i) by construction the result is fully compatible with quantum mechanics, (ii) there is full symmetry between preselected and postselected states, and (iii) it can easily incorporate any other constraint or information known about the system (Sec. $\mathrm{V})$.

This offers some advantages over previous formulations of the problem [1-3] (Secs. III and IV). The approach in Refs. $[1,2]$ addressing the statistics of intermediate measurements does not provide a legitimate quantum state supporting such statistics. The purely predictive or retrodictive approaches [3] lack symmetry between preselection and postselection.

\section{UNCONSTRAINED PREDICTION-RETRODICTION}

In this section we apply the maximum likelihood strategy to determine the legitimate quantum state that best describes the system at times $t$ between two measurements performed at times $t_{1}, t_{2}$ with $t_{1} \leq t \leq t_{2}$. In this section we assume that the only information about the system is the one provided exclusively by the two measurements. Additional constraints will be considered in Sec. V.

For definiteness and simplicity we will consider the usual case where the statistics of the measurements are given by projection on pure states. More specifically, we consider the

*alluis@ fis.ucm.es; http://www.ucm.es/info/gioq/alfredo.html probabilities $P_{j}=\left\langle\psi_{j}|\rho| \psi_{j}\right\rangle$ for $j=1,2$, where $\rho$ is the unknown state of the system at time $t$ and the vectors $\left|\psi_{j}\right\rangle$ subsume the evolution between $t_{1}, t$, and $t_{2}$ as (see Fig. 1)

$$
\left|\psi_{1}\right\rangle=U_{1}\left|\phi_{1}\right\rangle, \quad\left|\psi_{2}\right\rangle=U_{2}^{\dagger}\left|\phi_{2}\right\rangle
$$

where $U_{1}$ represents the forward evolution from $t_{1}$ to $t, U_{2}^{\dagger}$ represents the backward evolution from $t_{2}$ to $t$, and $\left|\phi_{1,2}\right\rangle$ represent the observed states at $t_{1,2}$. This is to say that $\left|\psi_{1}\right\rangle$ represents the predictive state as the forward evolution of the preselected state $\left|\phi_{1}\right\rangle$, while $\left|\psi_{2}\right\rangle$ represents the retrodictive state as the backward evolution of the postselected state $\left|\phi_{2}\right\rangle$.

From a Bayesian perspective we may consider that $\rho(t)$ is given by a weighted sum of all density matrices $\rho$ [4-7],

$$
\rho(t)=\frac{\sum_{\rho} P\left(\psi_{1}, \psi_{2} \mid \rho\right) \rho}{\sum_{\rho^{\prime}} P\left(\psi_{1}, \psi_{2} \mid \rho^{\prime}\right)},
$$

where the weight $P\left(\psi_{1}, \psi_{2} \mid \rho\right)$ is the likelihood function or conditional probability of obtaining the outcomes $\left|\psi_{1,2}\right\rangle$ in the two measurements when the state of the system is $\rho$,

$$
P\left(\psi_{1}, \psi_{2} \mid \rho\right)=P_{1} P_{2} \text {. }
$$

The likelihood function is commonly used as representing the information provided by multiple measurements [4-8]. Note that in Eq. (2.3) the density matrix appears twice. This is because there are two measurements and each measurement carries a dependence on the state of the system. For the sake of simplicity we will consider a maximum likelihood strategy by finding for each time $t$ the $\rho$ with the largest likelihood function $P_{1} P_{2}$. This is the state that maximizes the probability of obtaining the observed results.

The case $\left|\psi_{1}\right\rangle=\left|\psi_{2}\right\rangle$ is trivial since the optimum state will be $\rho=\left|\psi_{1}\right\rangle\left\langle\psi_{1}|=| \psi_{2}\right\rangle\left\langle\psi_{2}\right|$ with maximum probability $P_{1}=P_{2}$ $=1$. From now on we assume $\left|\psi_{1}\right\rangle \neq\left|\psi_{2}\right\rangle$.

Since we want to maximize the overlap of $\rho$ with $\left|\psi_{1,2}\right\rangle$ we can restrict ourselves to pure states $\rho=|\psi\rangle\langle\psi|$ lying in the two-dimensional subspace spanned by $\left|\psi_{1,2}\right\rangle$. Let us consider

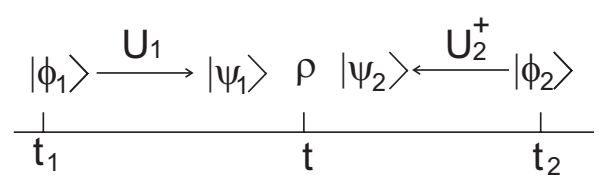

FIG. 1. Illustration of the relations between states $\left|\phi_{1,2}\right\rangle$ and $\left|\psi_{1,2}\right\rangle$. 
first that $\left\langle\psi_{1} \mid \psi_{2}\right\rangle \neq 0$. Without loss of generality we can adjust the phases of $\left|\psi_{1,2}\right\rangle$ so that $\eta=\left\langle\psi_{1} \mid \psi_{2}\right\rangle$ is a positive real number $\eta>0$. In such a case the following states are orthonormal:

$$
\left|\psi_{ \pm}\right\rangle=\frac{1}{\sqrt{2(1 \pm \eta)}}\left(\left|\psi_{1}\right\rangle \pm\left|\psi_{2}\right\rangle\right) .
$$

The most general pure state in the subspace spanned by $\left|\psi_{1,2}\right\rangle$ is $|\psi\rangle=\alpha_{+}\left|\psi_{+}\right\rangle+\alpha_{-}\left|\psi_{-}\right\rangle$with $\left|\alpha_{+}\right|^{2}+\left|\alpha_{-}\right|^{2}=1$. Since

$$
\left\langle\psi_{1,2} \mid \psi\right\rangle=\alpha_{+} \sqrt{\frac{1+\eta}{2}} \pm \alpha_{-} \sqrt{\frac{1-\eta}{2}},
$$

we have

$$
P_{1,2}=\frac{1+\eta}{2}\left|\alpha_{+}\right|^{2}+\frac{1-\eta}{2}\left|\alpha_{-}\right|^{2} \pm\left|\alpha_{+} \alpha_{-}\right| \sqrt{1-\eta^{2}} \cos \varphi,
$$

where $\varphi=\arg \alpha_{+}-\arg \alpha_{-}$. By direct computation of $P_{1} P_{2}$ and taking into account that $\eta>0$ it can be seen that the maximum $P_{1} P_{2}$ arises for $\alpha_{-}=0$ so that the maximum likelihood state is $|\psi\rangle=\left|\psi_{+}\right\rangle$,

$$
|\psi\rangle=\frac{1}{\sqrt{2(1+\eta)}}\left(\left|\psi_{1}\right\rangle+\left|\psi_{2}\right\rangle\right)=\frac{1}{\sqrt{2(1+\eta)}}\left(U_{1}\left|\phi_{1}\right\rangle+U_{2}^{\dagger}\left|\phi_{2}\right\rangle\right),
$$

with $P_{1}=P_{2}=(1+\eta) / 2$.

On the other hand, when $\left\langle\psi_{1} \mid \psi_{2}\right\rangle=0$ the most general pure state in the subspace spanned by $\left|\psi_{1,2}\right\rangle$ is $|\psi\rangle=\alpha_{1}\left|\psi_{1}\right\rangle$ $+\alpha_{2}\left|\psi_{2}\right\rangle$ with $\left|\alpha_{1}\right|^{2}+\left|\alpha_{2}\right|^{2}=1$. A simple calculus after the direct computation of $P_{1} P_{2}$ leads to maximum $P_{1} P_{2}$ when

$$
|\psi\rangle=\frac{1}{\sqrt{2}}\left[\left|\psi_{1}\right\rangle+\exp (i \delta)\left|\psi_{2}\right\rangle\right],
$$

with $P_{1}=P_{2}=1 / 2$, where $\delta$ is an arbitrary phase.

We can appreciate that the maximum likelihood state $|\psi\rangle$ is a fully symmetrical superposition of the predictive and retrodictive states $\left|\psi_{1,2}\right\rangle$. This kind of state has appeared before in the literature as an intermediate state between two alternatives [9]. Moreover, for pure states $\rho=|\psi\rangle\langle\psi|$ we have $P_{1} P_{2}=\left|\left\langle\psi_{1}|\rho| \psi_{2}\right\rangle\right|^{2}$ so there is a close relation between maximum likelihood and the weak value $\rho_{w}=\left\langle\psi_{1}|\rho| \psi_{2}\right\rangle /\left\langle\psi_{1} \mid \psi_{2}\right\rangle$ of the observable $\rho$ between the preselected and postselected states $\left|\psi_{1,2}\right\rangle[10,11]$. This is $P_{1} P_{2}=\left|\left\langle\psi_{1} \mid \psi_{2}\right\rangle\right|^{2}\left|\rho_{w}\right|^{2}$. This is consistent with results of the Bayesian estimation addressed in Ref. [11] to determine the value of an observable between preselection and postselection. In this context, regarding $\rho$ as an observable we note that for pure states $\rho=|\psi\rangle\langle\psi|$ the likelihood function $P_{1} P_{2}=\left|\left\langle\psi_{1} \mid \psi\right\rangle\right|^{2}\left|\left\langle\psi_{2} \mid \psi\right\rangle\right|^{2}$ coincides with the numerator of the Aharonov-Bergmann-Lebowitz (ABL) rule (3.1) below.

\section{COMPARISON WITH OTHER APPROACHES}

The analysis of the problem in Ref. [1] leads to the following formula for the statistics of an observable $O$ at a time intermediate between $t_{1}$ and $t_{2}$ (ABL rule):

$$
P\left(o_{j}\right)=\frac{\left|\left\langle o_{j} \mid \psi_{1}\right\rangle\right|^{2}\left|\left\langle o_{j} \mid \psi_{2}\right\rangle\right|^{2}}{\sum_{k}\left|\left\langle o_{k} \mid \psi_{1}\right\rangle\right|^{2}\left|\left\langle o_{k} \mid \psi_{2}\right\rangle\right|^{2}}
$$

where $\left|o_{j}\right\rangle$ are the orthogonal states determining the statistics of $O$ via projection, assumed pure for simplicity.

In our context the key point is that the statistics $P\left(o_{j}\right)$ is not supported by the existence of a legitimate quantum state. In the next section we illustrate this point with an example. Otherwise, the ABL rule is symmetric in the preselected and postselected states. The probabilities for the preselected (for $\left|o_{1}\right\rangle=\left|\psi_{1}\right\rangle$, and $\left\langle o_{j \neq 1} \mid \psi_{1}\right\rangle=0$ ) and postselected (for $\left|o_{1}\right\rangle$ $=\left|\psi_{2}\right\rangle$, and $\left.\left\langle o_{j \neq 1} \mid \psi_{2}\right\rangle=0\right)$ outcomes are according to Eq. (3.1) $P\left(\psi_{1}\right)=P\left(\psi_{2}\right)=1$.

On the other hand, for the purely predictive approach the state of the system at intermediate times is $|\psi\rangle=\left|\psi_{1}\right\rangle$ $=U_{1}\left|\phi_{1}\right\rangle$, where the probabilities $P_{1,2}$ are $P_{1}=\left|\left\langle\psi_{1} \mid \psi\right\rangle\right|^{2}=1$ and $P_{2}=\left|\left\langle\psi_{2} \mid \psi\right\rangle\right|^{2}=\eta^{2}$. For the purely retrodictive approach the state of the system at intermediate times is $|\psi\rangle=\left|\psi_{2}\right\rangle$ $=U_{2}^{\dagger}\left|\phi_{2}\right\rangle$, where the probabilities $P_{1,2}$ are $P_{1}=\left|\left\langle\psi_{1} \mid \psi\right\rangle\right|^{2}=\eta^{2}$ and $P_{2}=\left|\left\langle\psi_{1} \mid \psi\right\rangle\right|^{2}=1$.

It can be appreciated that in both cases the symmetry between preselection and postselection is lost, $P_{1} \neq P_{2}$. Moreover, the product $P_{1} P_{2}=\eta^{2}$ is lesser than the maximum likelihood value $P_{1} P_{2}=(1+\eta)^{2} / 4$ since $\eta<1$.

\section{EXAMPLE}

As the simplest illustrative example let us consider $\left|\psi_{1}\right\rangle$ $=|+z\rangle,\left|\psi_{2}\right\rangle=|+x\rangle$, where $| \pm j\rangle$ denote the eigenvectors of the Pauli matrices $\sigma_{j}, j=x, y, z$. We apply ABL rule (3.1) to the three observables $\sigma_{j}$ leading to

$$
\begin{aligned}
& P(+x)=1, \quad P(-x)=0, \\
& P(+y)=\frac{1}{2}, \quad P(-y)=\frac{1}{2}, \\
& P(+z)=1, \quad P(-z)=0 .
\end{aligned}
$$

These statistics cannot be derived from any quantum state $\rho$ since $P(+x)=P(+z)=1$ implies that $\rho$ should be eigenvector of $\sigma_{x}$ and $\sigma_{z}$ simultaneously.

The maximum likelihood approach provides the quantum state

$$
|\psi\rangle=\frac{1}{\sqrt{2+\sqrt{2}}}(|+z\rangle+|+x\rangle)
$$

leading to the statistics

$$
\begin{gathered}
P(+x)=\frac{3+2 \sqrt{2}}{4+2 \sqrt{2}}, \quad P(-x)=\frac{1}{4+2 \sqrt{2}}, \\
P(+y)=\frac{1}{2}, \quad P(-y)=\frac{1}{2},
\end{gathered}
$$




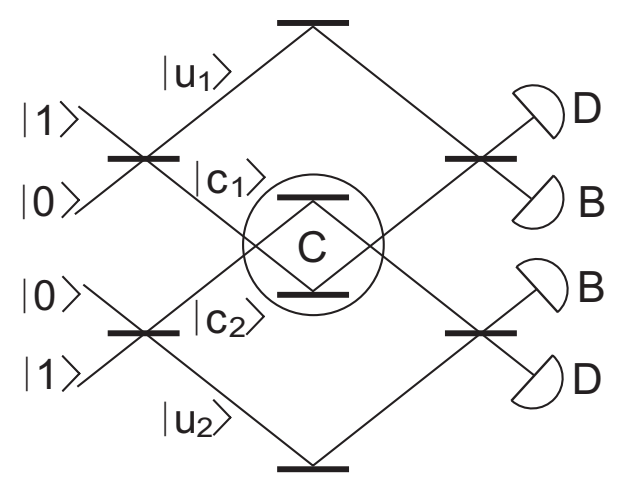

FIG. 2. Illustration of the Mach-Zehnder interferometers in the Hardy scheme.

$$
P(+z)=\frac{3+2 \sqrt{2}}{4+2 \sqrt{2}}, \quad P(-z)=\frac{1}{4+2 \sqrt{2}} .
$$

We can appreciate that this is quite similar to ABL rule (4.1). Let us develop this similarity further by showing that for this example the maximum likelihood solution (4.2) is the legitimate quantum state with mean values $\left\langle\sigma_{j}\right\rangle$ closest to the predictions of the ABL rule. To this end we compute the vector $\boldsymbol{r}$ with components $r_{j}=\left\langle\sigma_{j}\right\rangle_{A B L}=P(+j)-P(-j)$ for $P( \pm j)$ in Eq. (4.1) leading to $\boldsymbol{r}=(1,0,1)$. Let $\boldsymbol{s}$ be the vector $s_{j}=\left\langle\sigma_{j}\right\rangle$ for an arbitrary state. For legitimate quantum states $|\boldsymbol{s}| \leq 1$. Then, the vector $s$ closest to $\boldsymbol{r}$, i.e., with minimum $|\boldsymbol{r}-\boldsymbol{s}|$, is $\boldsymbol{s}=\boldsymbol{r} /|\boldsymbol{r}|=(1,0,1) / \sqrt{2}$ which corresponds precisely to the maximum likelihood state (4.2) as it can be checked from Eq. (4.3).

Finally, the predictive approach $|\psi\rangle=|+z\rangle$ leads to the statistics $P( \pm x)=1 / 2, P( \pm y)=1 / 2, P(+z)=1$, and $P(-z)=0$, while the retrodictive approach $|\psi\rangle=|+x\rangle$ leads to $P(+x)=1$, $P(-x)=0, P( \pm y)=1 / 2$, and $P( \pm z)=1 / 2$. It can be appreciated the lack of symmetry between preselection and postselection. Also, the probabilities are very different from the $\mathrm{ABL}$ rule and the maximum likelihood approach.

\section{CONSTRAINED LIKELIHOOD: HARDY SCHEME}

In this section we consider maximum likelihood optimization when there are extra restrictions on the set of admissible intermediate states. This is the case of the Hardy paradox [12], where two photons are incident in a pair of MachZehnder interferometers (see Fig. 2). Each interferometer is arranged so that in absence of perturbations the photon always leaves through the same exit port, the bright port B (the other is the dark port D).

One arm from each interferometer (the colliding arm) overlaps at the colliding region $\mathrm{C}$. It is assumed that if the photons coincide at the colliding region $\mathrm{C}$ they will be removed from the interferometer. This overlap perturbs the interference so there is some probability that the detectors at the dark ports click. This would indicate that the interference of the photon has been disturbed by the presence of the other photon in the colliding arm. This is an example of the socalled interaction-free detection [13].

The paradox arises when both photons are detected at the dark ports, since this seemingly would indicate that the two photons were simultaneously in the colliding arms. But in such a case they should have been removed from the interferometer, in contradiction with the fact that they were actually detected at the dark ports [12]. The source of the paradox is the combination of classical and quantum reasoning in a single argument, for example, invoking the presence or absence of the photons in the colliding arms as if they were exclusive possibilities.

Les us address this scheme from the point of view of the maximum likelihood approach. For convenience we express all states in terms the internal modes of the interferometer so that $\left|u_{j}\right\rangle$ and $\left|c_{j}\right\rangle$ represent the photon in the uncolliding or colliding arms, respectively. The evolved preparation state (predictive state) reads in these modes

$$
\left|\psi_{1}\right\rangle=\frac{1}{2}\left(\left|u_{1}\right\rangle+\left|c_{1}\right\rangle\right)\left(\left|u_{2}\right\rangle+\left|c_{2}\right\rangle\right),
$$

or $\quad\left|\psi_{1}\right\rangle=\frac{1}{2}\left(\left|u_{1}\right\rangle\left|u_{2}\right\rangle+\sqrt{2}|c u+\rangle+\left|c_{1}\right\rangle\left|c_{2}\right\rangle\right), \quad$ where $\quad|c u \pm\rangle$ $=\left(\left|c_{1}\right\rangle\left|u_{2}\right\rangle \pm\left|u_{1}\right\rangle\left|c_{2}\right\rangle\right) / \sqrt{2}$. The backward evolution of the $\left|\phi_{2}\right\rangle$ state associated with the simultaneous click of the two dark detectors (retrodictive state) is

$$
\left|\psi_{2}\right\rangle=\frac{1}{2}\left(\left|u_{1}\right\rangle-\left|c_{1}\right\rangle\right)\left(\left|u_{2}\right\rangle-\left|c_{2}\right\rangle\right),
$$

or $\left|\psi_{2}\right\rangle=\left(\left|u_{1}\right\rangle\left|u_{2}\right\rangle-\sqrt{2}|c u+\rangle+\left|c_{1}\right\rangle\left|c_{2}\right\rangle\right) / 2$. At difference with the analysis in Sec. II, in this case not all the state space spanned by $\left|\psi_{1,2}\right\rangle$ is available since the double click means that the probability that $|\psi\rangle$ is in the colliding state $\left|c_{1}\right\rangle\left|c_{2}\right\rangle$ vanishes. Thus, the optimization process must be carried out in terms of the state $|\psi\rangle=\alpha\left|u_{1}\right\rangle\left|u_{2}\right\rangle+\beta|c u+\rangle$, with $|\alpha|^{2}+|\beta|^{2}$ $=1$ and we have already taken into account that $\left|\psi_{1,2}\right\rangle$ have no projection on $|c u-\rangle$. Then we have

$$
P_{1,2}=\frac{1}{4}|\alpha \pm \sqrt{2} \beta|^{2} .
$$

By direct computation of $P_{1} P_{2}$ it can be easily seen that the maximum $P_{1} P_{2}$ occurs for $\alpha=0$ leading to the maximum likelihood state

$$
|\psi\rangle=|c u+\rangle=\frac{1}{\sqrt{2}}\left(\left|c_{1}\right\rangle\left|u_{2}\right\rangle+\left|u_{1}\right\rangle\left|c_{2}\right\rangle\right),
$$

with $P_{1}=P_{2}=1 / 2$. By construction the probability of collision is zero $P(c c)=\mid\left\langle c_{1}\left|\left\langle c_{2} \mid \psi\right\rangle\right|^{2}=0\right.$. The maximum likelihood state is entangled in sharp contrast to the purely predictive and retrodictive states in Eqs. (5.1) and (5.2) which are separable. This captures the quantum nature of the effect and the origin of the paradox since the entanglement in state (5.4) has no room in the classical language.

For the purely predictive and purely retrodictive approaches we have $P_{1}=1, P_{2}=0$ and $P_{1}=0, P_{2}=1$. In both cases there is a $25 \%$ probability of collision $P(c c)=1 / 4$ with both photons being removed from the interferometer.

Finally, when computing the probability of collision $P(c c)$ with the ABL rule the result depends on how the projector on $\left|c_{1}\right\rangle\left|c_{2}\right\rangle$ is embedded in a complete observable $O[1,2]$. For example, for the set $\left|o_{j}\right\rangle=\left\{\left|c_{1}\right\rangle\left|c_{2}\right\rangle,\left|u_{1}\right\rangle\left|u_{2}\right\rangle,|c u \pm\rangle\right\}$ we get 
$P(c c)=1 / 6 \quad[$ with $P(u c+)=2 / 3]$, while for the set $\left|o_{j}\right\rangle$ $=\left\{\left|c_{1}\right\rangle\left|c_{2}\right\rangle,\left|u_{1}\right\rangle\left|u_{2}\right\rangle,\left|u_{1}\right\rangle\left|c_{2}\right\rangle,\left|c_{1}\right\rangle\left|u_{2}\right\rangle\right\}$ we get $P(c c)=1 / 4$.

\section{DISCUSSION AND CONCLUSIONS}

We have addressed what can be said about a quantum system between preparation and observation. This has been done via a maximum likelihood strategy by determining the quantum state maximizing the probability of obtaining the observed outcomes.

The proper framework for this approach is the idea of quantum state as representing all our knowledge about the system. In our case this is the outcomes of the preselection and postselection measurements. Besides the quantum nature of these measurements, the relation with quantum mechanics stems from the fact that such inference must be compatible with quantum mechanics. This is the point where the ABL rule fails in the sense that it predicts probabilities that cannot be derived from any density matrix.

From an operational perspective, this kind of inference is very common in diverse areas, such as quantum metrology and quantum tomography [4-8], where the task is to retrieve as much information as possible of the system from available data, usually noisy and incomplete. In this regard we have shown that the maximum likelihood solution leads to legitimate quantum states with probabilities for intermediate measurements closest to the predictions of the ABL rule.

\section{ACKNOWLEDGMENTS}

This work was supported by Project No. FIS2008-01267 of the Spanish Dirección General de Investigación del Ministerio de Ciencia e Innovación.
[1] Y. Aharonov, P. G. Bergmann, and J. L. Lebowitz, Phys. Rev. 134, B1410 (1964); R. H. Penfield, Am. J. Phys. 34, 422 (1966); Y. Aharonov and D. Z. Albert, Phys. Rev. D 29, 223 (1984); D. Z. Albert, Y. Aharonov, and S. D’Amato, Phys. Rev. Lett. 54, 5 (1985); Y. Aharonov and L. Vaidman, J. Phys. A 24, 2315 (1991).

[2] O. Cohen, Phys. Rev. A 51, 4373 (1995); 56, 5191 (1997); L. Vaidman, ibid. 57, 2251 (1998).

[3] D. T. Pegg and S. M. Barnett, J. Opt. B: Quantum Semiclassical Opt. 1, 442 (1999); S. M. Barnett, D. T. Pegg, and J. Jeffers, J. Mod. Opt. 47, 1779 (2000).

[4] K. R. W. Jones, Ann. Phys. (N.Y.) 207, 140 (1991); Phys. Rev. A 50, 3682 (1994).

[5] Z. Hradil, Phys. Rev. A 55, R1561 (1997).

[6] R. Schack, T. A. Brun, and C. M. Caves, Phys. Rev. A 64, 014305 (2001).

[7] Quantum State Estimation, Springer Lecture Notes in Physics Vol. 649, edited by M. G. A. Paris and J. Řeháček (Springer, Berlin, 2004).

[8] V. Bužek, G. Adam, and G. Drobný, Ann. Phys. (N.Y.) 245, 37 (1996).
[9] A. Luis, Phys. Rev. A 67, 032108 (2003); B. Baseia, A. F. de Lima, and G. C. Marques, Phys. Lett. A 204, 1 (1995); A. F. De Lima, B. Baseia, and G. C. Marques, J. Mod. Opt. 43, 729 (1996).

[10] Y. Aharonov, D. Z. Albert, and L. Vaidman, Phys. Rev. Lett. 60, 1351 (1988); Y. Aharonov and L. Vaidman, Phys. Rev. A 41, 11 (1990); L. M. Johansen, Phys. Rev. Lett. 93, 120402 (2004); L. M. Johansen and A. Luis, Phys. Rev. A 70, 052115 (2004).

[11] L. M. Johansen, Phys. Lett. A 322, 298 (2004).

[12] L. Hardy, Phys. Rev. Lett. 68, 2981 (1992); W. T. M. Irvine, J. F. Hodelin, Ch. Simon, and D. Bouwmeester, ibid. 95, 030401 (2005); J. S. Lundeen and A. M. Steinberg, ibid. 102, 020404 (2009).

[13] A. C. Elitzur and L. Vaidman, Found. Phys. 23, 987 (1993); L. Vaidman, Quantum Opt. 6, 119 (1994); P. Kwiat, H. Weinfurter, T. Herzog, A. Zeilinger, and M. A. Kasevich, Phys. Rev. Lett. 74, 4763 (1995); P. Kwiat, H. Weinfurter, T. Herzog, A. Zeilinger, and M. Kasevich, Ann. N.Y. Acad. Sci. 755, 383 (1995); A. Luis and L. L. Sánchez-Soto, Phys. Rev. A 58, 836 (1998). 